\title{
Creutzfeldt-Jakob disease: updated diagnostic criteria, treatment algorithm, and the utility of brain biopsy
}

\author{
Marc Manix, MD, Piyush Kalakoti, MD, Miriam Henry, BS, Jai Thakur, MD, Richard Menger, MD, \\ Bharat Guthikonda, MD, and Anil Nanda, MD, MPH
}

Department of Neurosurgery, Louisiana State University Health Sciences Center, Shreveport, Louisiana

Creutzfeldt-Jakob disease (CJD) is a rare neurodegenerative condition with a rapid disease course and a mortality rate of $100 \%$. Several forms of the disease have been described, and the most common is the sporadic type. The most challenging aspect of this disease is its diagnosis-the gold standard for definitive diagnosis is considered to be histopathological confirmation - but newer tests are providing means for an antemortem diagnosis in ways less invasive than brain biopsy. Imaging studies, electroencephalography, and biomarkers are used in conjunction with the clinical picture to try to make the diagnosis of CJD without brain tissue samples, and all of these are reviewed in this article. The current diagnostic criteria are limited; test sensitivity and specificity varies with the genetics of the disease as well as the clinical stage. Physicians may be unsure of all diagnostic testing available, and may order outdated tests or prematurely request a brain biopsy when the diagnostic workup is incomplete. The authors review CJD, discuss the role of brain biopsy in this patient population, provide a diagnostic pathway for the patient presenting with rapidly progressive dementia, and propose newer diagnostic criteria.

http://thejns.org/doi/abs10.3171/2015.8.FOCUS15328

KEY WORDS Creutzfeldt-Jakob disease; prion; brain biopsy; diagnostic criteria; diagnostic algorithm; National (Nationwide) Inpatient Sample

$\mathrm{T}$ HE term Creutzfeld-Jakob disease (CJD) was first used in 1922 by Spielmeyer to name the condition described by 2 German physicians. ${ }^{48}$ The 6 original subjects (a case report by Creutzfeld and a series of 5 patients by Jakob) were a heterogeneous group of patients with unusual neuropathological findings associated with other disorders, and years later only 2 of the cases were confirmed with modern techniques to actually be CJD. ${ }^{14,22,33}$ In 1960 the typical clinical picture, electroencephalography (EEG) findings, and classic spongiform changes in the neuropil were recognized as the cardinal features of the disease. ${ }^{38}$

Now there are diagnostic criteria to guide the physician in the workup of a patient with suspected CJD; physical examination and tests are used to provide a definitive, probable, or possible diagnosis. A brain biopsy is able to give pathological confirmation of the disease and is considered the gold standard for diagnosis. However, research has progressed to the point where newer tests should be considered in the diagnostic criteria and may be more sensitive in disease confirmation than tissue obtained from surgery.

Creutzfeld-Jakob disease is classified as familial, sporadic, or acquired. Regardless of the type, the disease has a rapid clinical course that is uniformly fatal. There are some consistencies on physical examination, radiographic studies, and EEG, but the most common form is sporadic CJD (sCJD), and it follows a theme of heterogeneity. The infectious agent is the abnormal scrapie form $\left(\mathrm{PrP}^{\mathrm{Sc}}\right)$ of the host-encoded cellular prion protein $\left(\mathrm{PrP}^{\mathrm{C}}\right)$ that causes a posttranslational modification of $\mathrm{PrP}^{\mathrm{C}}$ into the disease form, accumulating in the brain and causing neurodegeneration. Familial CJD (fCJD), or the genetic type, is due to a mutation in the gene encoding $\operatorname{PrP}^{\mathrm{C}}$, whereas the sporadic form is thought to originate after a somatic mutation or a stochastic protein alteration. Creutzfeld-Jakob disease is

ABBREVIATIONS ADC = apparent diffusion coefficient; BSE = bovine spongiform encephalopathy; CDI = conformation-dependent immunoassay; CJD, fCJD, sCJD, vCJD = Creutzfeld-Jakob disease, familial CJD, sporadic CJD, variant CJD; DWI = diffusion-weighted imaging; EEG = electroencephalography; FFI = fatal familial insomnia; GSS = Gerstmann-Sträussler-Scheinker; NIS = National (Nationwide) Inpatient Sample; PSWCs = periodic sharp wave complexes; RT-QuIC = real-time quaking-induced conversion; UK = United Kingdom.

SUBMITTED July 1, 2015. ACCEPTED August 6, 2015.

INCLUDE WHEN CITING DOI: 10.3171/2015.8.FOCUS15328. 
also transmissible through iatrogenic causes or by ingesting beef with bovine spongiform encephalopathy (BSE or "mad cow disease"), leading to variant CJD (vCJD). ${ }^{25}$

Creutzfeld-Jakob disease is rare, and is often a diagnostic challenge for physicians facing a rapidly progressing dementia. As of now, definitive diagnosis is provided with brain biopsy, but more often than not the results are inconclusive because not all areas of the brain will show the classic histological changes in CJD, even if the disease is present. Surgeons target areas that appear the most abnormal on imaging studies, but this is most often in deepseated subcortical structures. The WHO criteria, as well as more updated suggested criteria, are outdated because they do not use newer tests that provide a less invasive method for definitive diagnosis. Given these issues, a newer approach to diagnosis is needed. In this paper we briefly review the disease, assess the current tools used in making the diagnosis, discuss when a brain biopsy should be performed, propose changes to diagnostic criteria, and provide an algorithm for the workup of the patient with a rapidly progressive dementia of unknown origin.

\section{Pathophysiology}

To understand some of the clinical findings of the disease, one must first review the pathophysiology of how a healthy protein becomes abnormal and destructive to the brain. $\mathrm{PrP}^{\mathrm{C}}$ is found in lipid rafts on the cell surface of normal brains. The function of the protein is unknownprion protein (PRNP) knockout mice completely lacking the protein do not show any obvious abnormalities, and have normal brain development.?

The central pathological event is the formation of the abnormal PrPsc from the wild-type, cellular form of $\mathrm{PrPC}^{\mathrm{C}}$. This is hypothesized to occur in a pathway where $\mathrm{PrP}^{\mathrm{Sc}}$ serves as the template for $\mathrm{PrP}^{\mathrm{C}}$ to fold abnormally into the pathogenic conformation. This is an autocatalytic process that is poorly understood, but the change in protein shape is the hallmark of the pathology. ${ }^{44}$

Both forms have an identical amino acid sequence (primary structure), but the posttranslational changes cause the $\operatorname{PrP}^{\mathrm{C}}(40 \%$ alpha helix) to refold into a form with $45 \%$ beta-sheet composition. ${ }^{44}$ This makes the protein not only highly insoluble, but also resistant to proteinase digestion. The subsequent multimerization accumulates, spreads throughout the brain parenchyma, and induces the classic spongiform change (vacuolation of gray matter) by microglial activation and neuronal loss, leading to progressive neurodegeneration and astrogliosis over time.

\section{Genetics}

$\mathrm{PrP}^{\mathrm{C}}$ is encoded by the prion protein $(P R N P)$ gene on human chromosome $20 .{ }^{29}$ All familial forms of the disease are characterized by a mutation in this gene. PRNP has a normal genetic polymorphism at codon 129 , where either methionine (M) or valine (V) may be encoded. This is very important because the genotype imparts genetic susceptibility in all types of prion disease. The most dramatic example of this is that all cases of VCJD to date are in individuals homozygous for methionine (MM) ${ }^{16}$

Parchi et al. ${ }^{41}$ classified sCJD into 6 different molecular strains based on the genotype and biochemical properties. When $\mathrm{PrP}^{\mathrm{Sc}}$ is cleaved by proteinase $\mathrm{K}$ under defined conditions, there are 2 truncated forms that result: the $21-\mathrm{kD}$ fragment (Type 1) and one that is $19 \mathrm{kD}$ (Type 2). The importance of this classification into MM1, MM2, MV1, MV2, VV1, and VV2 is that the different strains strongly correlate with different pathologically distinct phenotypes, which is summarized in Table 1. The fact that $\mathrm{PrP}^{\mathrm{Sc}}$ has the same amino acid sequence but different pathological and clinical presentation implies that the conformational variant is what determines the phenotypic or molecular "strain."

The changes in protein structure are investigated by a conformation-dependent immunoassay (CDI). This test identifies $\mathrm{PrP}^{\mathrm{Sc}}$ by exposing specific epitopes of the protein that are unmasked with progressive denaturation, and antibodies specific to these areas bind and elicit a positive result. It is $100 \%$ specific for the disease, and recent data show sensitivity that is at least equal to other diagnostic tests.

\section{Forms of CJD Sporadic CJD}

By far the most common form of the disease is the sporadic type-SCJD-which occurs at an incidence of 1 case in 1 million per annum, and accounts for $85 \%$ of CJD cases. Researchers speculate that it results from a spontaneous neurodegenerative illness, and the hypothesis is that it results from either a somatic mutation in the gene or a random structural change in the PrP protein causing formation of $\mathrm{PrP}^{\mathrm{Sc}}$. Onset usually occurs in the 7th decade of life, and the median time to death is 5 months, with $90 \%$ of patients dead by 1 year. ${ }^{23}$ Unlike vCJD, the clinical and pathological findings are more heterogeneous, and this is probably due to the different molecular phenotypes present.

\section{Familial CJD}

Familial CJD is a result of known mutations of PRNP. This accounts for approximately $10 \%$ of all cases of prion diseases $^{35}$ and is historically split into the 3 phenotypic categories of Gerstmann-Sträussler-Scheinker (GSS) syndrome, fatal familial insomnia (FFI), and fCJD. There are more than 50 mutations described, and the disease is transmitted in an autosomal dominant pattern with high penetrance, and with an incidence that increases with age. fCJD has similar clinical, radiographic, and test findings as SCJD, whereas GSS and FFI are well-described variants. ${ }^{35}$ A comparison of the familial types with sporadic and variant CJD is provided in Table 2.

\section{latrogenic CJD}

Creutzfeld-Jakob disease can be acquired iatrogenically; several outbreaks over the last few decades were associated with intracerebral electrodes, corneal transplantation, dura mater grafts, and growth hormone injections. The first case report, in 1974, was in a patient who received a corneal transplant from an infected cadaver. ${ }^{15}$ Dura mater grafts with contaminated material led to more than 60 cases of CJD, with incubation periods lasting be- 
TABLE 1. Genetic subtypes of SCJD and typical features

\begin{tabular}{|c|c|c|c|c|c|}
\hline \multirow[b]{2}{*}{ Features } & \multicolumn{5}{|c|}{ sCJD Subtype } \\
\hline & MM1/MV1 & VV2 & MV2 & MM2 & VV1 \\
\hline Mean age at onset & 70 yrs & $65 \mathrm{yrs}$ & 60 yrs & 67 yrs & 44 yrs \\
\hline Duration of illness & $4 \mathrm{mos}$ & $6 \mathrm{mos}$ & $18 \mathrm{mos}$ & 14 mos & $21 \mathrm{mos}$ \\
\hline $\begin{array}{l}\text { Predominant clinical } \\
\text { features }\end{array}$ & $\begin{array}{l}\text { Rapid cognitive decline } \\
\text { w/ myoclonus }\end{array}$ & $\begin{array}{l}\text { Progressive ataxia in } \\
\text { absence of myoc- } \\
\text { lonus }\end{array}$ & $\begin{array}{l}\text { Prominent ataxia \& } \\
\text { cognitive decline }\end{array}$ & $\begin{array}{l}\text { Rapidly progressive de- } \\
\text { mentia w/ myoclonus }\end{array}$ & $\begin{array}{l}\text { Psychiatric changes, } \\
\text { slowly progressive } \\
\text { dementia }\end{array}$ \\
\hline MRI findings & $\begin{array}{l}70 \% \text { MRI hyperintensity } \\
\text { in basal ganglia or } \\
\text { cortex }\end{array}$ & $\begin{array}{l}70 \% \text { hyperintensity in } \\
\text { basal ganglia, } 45 \% \text { in } \\
\text { thalamus }\end{array}$ & $\begin{array}{l}79 \% \text { hyperintensity } \\
\text { in basal ganglia, + } \\
\text { pulvinar sign }\end{array}$ & $\begin{array}{l}\text { Cortical changes in } \\
25 \% \text {, rare basal } \\
\text { ganglia involvement }\end{array}$ & $\begin{array}{c}\text { Frequent cortical hyper- } \\
\text { intensity, rare basal } \\
\text { ganglia involvement }\end{array}$ \\
\hline EEG findings & PSWCs in $80 \%$ & PSWCs in $10 \%$ & Similar to VV2 & PSWCs in $42 \%$ & PSWCs negative \\
\hline 14-3-3 status & 95\% positive & $80 \%$ positive & Similar to VV2 & 91\% positive & $\begin{array}{l}\text { Positive in nearly all } \\
\text { cases }\end{array}$ \\
\hline $\begin{array}{l}\text { Percentage of SCJD } \\
\text { cases }\end{array}$ & $60 \%-70 \%$ & Approximately $15 \%$ & Approximately $10 \%$ & Approximately $5 \%$ & Approximately $1 \%$ \\
\hline
\end{tabular}

tween 1 and 14 years..$^{10}$ Pooled cadaveric growth hormone was used for injections and led to CJD 5-30 years after the injections. The clinical symptoms are similar to sCJD, as are the MRI and EEG findings. ${ }^{6}$ Iatrogenic CJD also has a clinical picture that is similar to SCJD.

It is clear that the incubation time is reflective of the inoculation site. Those with contaminated electrodes placed directly in the brain had short incubation periods of 16-28 months, whereas peripheral injections of growth hormone took anywhere from 5 to 30 years for the symptoms to begin. ${ }^{52}$ There are 3 cases of probable transmission of CJD to individuals who received blood transfusions from a donor with vCJD, which is why there is a ban on donors who lived in the United Kingdom (UK) during the epidemic of BSE. ${ }^{30}$

\section{Variant CJD}

Variant CJD was first described in 1996 and is the result of eating food contaminated with BSE, which was a major problem in the late 1980s through the early 1990s in the UK. ${ }^{56}$ Since the original 1996 report, there have been a total of 229 confirmed cases worldwide, with the most being found in the UK. There have been 4 total cases in the US, with 3 of the 4 having a history of residence in the UK. ${ }^{32}$
The clinical and pathological findings differ from other types of CJD (Table 2), in that the early course consists of mostly psychiatric symptoms before ataxia begins at approximately the 6-month mark. The median age of onset is much younger than in the sporadic or familial types, and the mean survival is also longer at 14 months. It is unclear if the survival increase is due to the age differences, but recent epidemiological studies do suggest that exposure alone is not sufficient to explain the higher incidence, and that age is an important risk factor for contracting the disease. ${ }^{4}$ Again, genetics play a crucial role; all confirmed cases were homozygous for methionine (MM) at the polymorphic codon 129 of the PRNP gene.

As with peripheral exposure to the infectious protein in acquired CJD, the incubation period is longer in vCJD given the parenteral route of infection. Most patients who developed the disease were exposed in the late 1980s, and the peak incidence of vCJD was in the early 2000s, giving an incubation period of 11-12 years. Another distinct feature is that $\mathrm{PrP}^{\mathrm{Sc}}$ accumulates in germinal follicles and lymph nodes, and has been pathologically confirmed after tonsillectomy. This deposition of the abnormal protein in the lymphoreticular system is not seen in other human prion diseases. ${ }^{21}$

TABLE 2. Findings in different types of CJD

\begin{tabular}{|c|c|c|c|c|c|}
\hline \multirow[b]{2}{*}{ Features } & \multicolumn{5}{|c|}{ CJD Type } \\
\hline & sCJD & vCJD & fCJD & GSS & $\mathrm{FFI}$ \\
\hline Mean age at onset & $60-70$ yrs & $28 \mathrm{yrs}$ & 60 yrs & $60 \mathrm{yrs}$ & 50 yrs \\
\hline Duration of illness & $5 \mathrm{mos}$ & 14 mos & $6 \mathrm{mos}$ & 5 yrs & 14 mos \\
\hline $\begin{array}{l}\text { Predominant clinical } \\
\text { features }\end{array}$ & $\begin{array}{l}\text { Rapid cognitive decline, myo- } \\
\text { clonus }\end{array}$ & $\begin{array}{l}\text { Early psychiatric symptoms, } \\
\text { then cognitive decline }\end{array}$ & Similar to SCJD & Cerebellar signs & Insomnia \\
\hline MRI findings & $\begin{array}{c}60 \%-70 \% \text { have hyperintesity } \\
\text { in basal ganglia or cortex }\end{array}$ & Pulvinar sign in $90 \%$ & $\begin{array}{l}\text { Basal ganglia \& cortical } \\
\text { hyperintensity }\end{array}$ & Rarely abnormal & $\begin{array}{l}\text { Nonspecific } \\
\text { atrophy }\end{array}$ \\
\hline EEG findings & PSWCs in $60 \%-70 \%$ & PSWCs negative & PSWCs in $75 \%$ & Rarely positive & Rarely positive \\
\hline $14-3-3$ status & Positive in $90 \%$ & Positive in $50 \%$ & Similar to SCJD & Negative & Rarely positive \\
\hline Genetics & MM1 most common (70\%) & MM in $100 \%$ & PRNP mutation & $\begin{array}{l}\mathrm{P} 102 \mathrm{~L} \text { is most com- } \\
\text { mon mutation }\end{array}$ & D178N mutation \\
\hline
\end{tabular}


Pathological investigation shows the characteristic spongiform change with gliosis in the brain, predominantly in the basal ganglia and cerebellum. The most severe damage is in the pulvinar, which correlates with the characteristic high signal abnormality on T2 MRI studies that is seen in $75 \%$ of cases. In contrast to the familial and sporadic types, the EEG study is usually negative for any periodic sharp wave forms. ${ }^{21}$

\section{Molecular Strains}

The most common strain is the MM1/MV1. These two are combined because there are no differences in the pathological features, and there is similarity between the clinical symptoms. Between $60 \%$ and $70 \%$ of all CJD cases are grouped here, with $95 \%$ of these being MM1. These patients fall under the "classic" CJD criteria, with myoclonus, an age of onset in the 7th decade of life, and a short mean disease duration of 4 months. The most common presentation is cognitive impairment, but prominent visual signs (Heidenhain's variant), cerebellar ataxia, and psychiatric symptoms can be present as well. The main difference between MM1 and MV1 is that in patients with MV1, ataxia is the more prominent neurological finding over cognitive impairment, especially early in the disease course. EEG is positive for periodic sharp wave complexes (PSWCs) in the first 3 months (80\%), and 14-3-3 protein sensitivity is $95 \% .{ }^{41}$ MRI shows changes in the striatum and cerebral cortex in $70 \%$ of cases. ${ }^{16} \mathrm{VV} 2$ is the next most common; it is seen in approximately $15 \%$ of cases. There is a similar age of onset at 65 years and a slightly longer clinical course of 6 months, but EEG is nonspecific and rarely shows PSWCs (less than 10\% of cases). MRI again shows hyperintensity in the striatum and thalamus, and the sensitivity of the $14-3-3$ protein assay is $80 \%{ }^{41}$ This group can be distinguished on examination because of the predominance of rapidly progressive ataxia in the absence of myoclonus, which does not appear until late.

MV2 is present in $9 \%$ of the patient population and is distinguished from all other subtypes because of the significantly longer disease course. There is a mean age of onset at 60 years, but the duration of the disease is, on average, 18 months. This slower progression rate is in conjunction with prominent ataxia as well as the more typical cognitive decline, myoclonus, and psychiatric signs. The EEG and 14-3-3 results are similar to those in VV2, but the MRI shows thalamic signal most frequently in the pulvinar, leading to the described "pulvinar sign." 27

MM2 also has a longer disease course (14 months), and is seen in $2 \%-8 \%$ of cases. The main difference between MM2 and MV2 is that there is mostly cognitive impair- ment in MM2, as opposed to the predominant ataxia in the latter. EEG shows PSWCs in 42\%, and MRI has increased cortical signal in $25 \%$, usually seen in the temporal lobe and with little basal ganglia involvement..$^{26,42}$

The final group, the VV1, is the most uncommon, being seen in just $1 \%$ of cases. This is an early-onset subtype, with the reported age of onset being 40-44 years, and a disease duration of 21 months. A prominent, slowly progressive frontotemporal dementia with psychiatric changes is described; MRI shows hyperintensity in the cortex without much basal ganglia involvement, EEG does not show PSWCs, but the test for 14-3-3 protein is almost always positive..$^{36,42}$

\section{Clinical Signs}

The hallmark of CJD is rapidly progressive dementia of unknown origin. Also, numerous atypical neurological examination findings are commonly seen: myoclonus, visual changes leading to cortical blindness, ataxia, and usually an akinetic mutism in the last stages of the disease. Myoclonus is the most common sign, but there are atypical findings such as sleep disturbances, chorea, psychiatric symptoms, and peripheral neuropathy. ${ }^{19}$ The clinical examination can highlight the heterogeneity of CJD phenotypes, but the common factor is progressive decline in neurological status. The most common physical examination findings stratified by phenotypic strain are listed in Table 1 .

\section{Diagnostic Tests}

In 1998 the WHO published diagnostic criteria for CJD (Table 3), with the diagnosis relying on clinical examination, EEG, and CSF findings. This is somewhat outdated in contemporary medicine because it does not take into account MRI findings, genetic testing, or modern laboratory tests for confirmation of the diagnosis. EEG and testing for 14-3-3 protein in the CSF are included in the criteria, and these studies are commonly done in the early stages of the diagnostic workup.

\section{EEG Findings}

Periodic sharp wave complexes (PSWCs) are found in the EEG recordings of approximately two-thirds of patients with SCJD, and have therefore been incorporated into probable sCJD diagnostic criteria by the WHO. ${ }^{55}$ The typical appearance for SCJD is that of a $1 /$ second periodic triphasic sharp wave complex. The simple sharp waves can be classic triphasic, biphasic, or mixed. EEG-related spikes are independent from the traditional clinical finding of myoclonic jerking and are more likely to be related

TABLE 3. The 1998 WHO diagnostic criteria for CJD*

\begin{tabular}{|c|c|c|}
\hline A & $\mathrm{B}$ & C \\
\hline \multirow{4}{*}{ Rapidly progressive dementia } & Myoclonus & \multirow{2}{*}{ PSWCs on EEG during an illness of any duration } \\
\hline & Visual or cerebellar signs & \\
\hline & Pyramidal/extrapyramidal signs & \multirow{2}{*}{$\begin{array}{l}\text { Positive } 14-3-3 \text { CSF assay in patients w/ disease duration } \\
\text { of }<2 \text { yrs }\end{array}$} \\
\hline & Akinetic mutism & \\
\hline
\end{tabular}


to the fusion of dendritic membranes in neurons. This subsequently results in electronic coupling through synchronization in the corpus callosum. ${ }^{51}$

Historically, PSWCs for CJD were described as typically presenting in a bilateral frontal midline. However, there is growing evidence to support a more temporal EEG diagnostic template among patients diagnosed with SCJD. A lateralized PSWC distribution represents the prodromal stage of disease onset. This localization will then progress to the typical more global bifrontal findings as the disease accelerates..$^{54}$ This has dramatic effects on the clinical use of EEG findings as diagnostic criteria during initial disease onset.

Although lateralized PSWCs are described early in the disease course, nonspecific diffuse slowing background EEG patterns or other nondescriptive findings for primary cerebral function are most common. The sensitivity of EEG is generally low in SCJD, but when positive it is of value, giving a very high specificity. Heinemann et al. looked at PSWCs at a CJD surveillance unit in Germany, and found $37.5 \%$ sensitivity and $100 \%$ specificity in their cohort of 26 patients. ${ }^{20}$ When combined with clinical presentation, examination, and symptoms, the positive predictive value of diagnosis reached $99 \%$.

On average, there is a 3.7-month delay from disease onset to the manifestation of PSWC findings on EEG. This is temporally limited by the fact that there is only a mean 8-week survival after development of PSWCs with CJD. Even more important is that not all patients with sCJD develop PSWCs; these complexes are typically found in patients who have the MV1 and MM1 genotypes and not in those with valine homozygous variant at codon 129.58 The false-positive EEG findings were found in patients with Alzheimer disease and vascular dementia-patients who can easily be differentiated based on their profoundly different clinical disease presentation. ${ }^{54}$

It is vital to recognize that EEG patterns associated with CJD are sensitive to disease timing, benzodiazepines, and external stimulation. The PSWCs are likely to present while patients are awake and to be exacerbated with sleep deprivation. Benzodiazepines mask PSWC findings on EEG for patients with sCJD. Seizures, especially status epilepticus treated with benzodiazepines, are typically found in $15 \%$ of patients with CJD and may cloud the utility of EEG in diagnosis.

\section{MRI Findings}

Initial studies investigating the usefulness of the MRI in the diagnosis of CJD used a T2 imaging pattern consistently. ${ }^{3}$ The technological advancements in MRI enabled physicians to use FLAIR, diffusion-weighted imaging (DWI), and apparent diffusion coefficient (ADC), improving both the negative and positive predictive value. ${ }^{8}$ The term "pulvinar sign," or bilateral FLAIR hyperintensity of the pulvinar area, was coined after reviewing 86 patients with vCJD. In that study $90 \%$ of the MR images were positive for the sign. ${ }^{13}$ However, recent comparative studies reliably concluded that both DWI and FLAIR were comparable in detecting hyperintensities in basal ganglia, although DWI was clearly superior in detection of similar changes in the neocortex. ${ }^{24}$
Now the anatomical areas of diagnosis have expanded from the traditional basal ganglia/thalamic areas to frontal, parietal, visual, temporal, limbic, and hippocampal cortices, which also correlate to the different molecular types of CJD. The pathophysiology of CJD with respect to imaging pattern is unique. Astrogliosis has been linked to high-intensity signal patterns on T2/FLAIR sequences. Restricted diffusion of water leading to hyperintensity signals in DWI is attributed to the vacuole formation and deposition of prion protein in CJD, and is accounted as the principal neuropathological finding in the latter. ${ }^{37}$

Hyperintensity on DWI and ADC studies also correlate with the symptoms and clinical course of CJD. In patients with hyperintensity of basal ganglia on DWI, there is a shorter disease duration and higher incidence of myoclonus. Shorter time between symptom onset and akinetic mutism is strongly correlated with the patients having hyperintense lesions in the occipital cortex on DWI. ${ }^{17}$

Diffusion tensor imaging was recently incorporated to study the pathogenesis of CJD in relation to white matter. Significant reductions of fractional anisotropy in patients with CJD in distinct and functionally relevant white matter pathways (including corticospinal, internal capsule, external capsule, fornix, and posterior thalamic radiation) correlate with progressive leukoencephalopathy and, more importantly, provide diagnostic criteria early in the disease course. ${ }^{28}$

The disease stage also has a significant impact on the nature of the MRI pattern. DWI is considered superior to any other MRI sequence in the early stages of CJD. ${ }^{53} \mathrm{Hy}-$ perintensity decreases in the later stages of the disease, and the only findings may be cortical atrophy. ${ }^{31}$ This is crucial information for the clinician to remember when the possibility of CJD is in the differential diagnosis, because the stage of the disease in relation to the positive predictive value of diagnostic criteria must be considered.

Patterns of FLAIR/DWI hyperintensity and restricted diffusion have also been shown to differentiate sporadic CJD from other causes of rapidly progressive dementia. Vitali et al. studied these MRI sequences across 90 patients, and found gray matter hyperintensities in all cases of SCJD, but never confined to just limbic regions. ${ }^{53} \mathrm{In}$ all SCJD cases with basal ganglia or thalamic DWI hyperintensities, there was associated restricted diffusion in the ADC map. These ADC findings were not seen in any nonprion-related cases, where isolated limbic hyperintensities (FLAIR > DWI) were common. Combined MRI sensitivity and specificity for SCJD was $96 \%$ and $93 \%$, respectively, and these investigators concluded that the pattern of FLAIR/DWI hyperintensity and restricted diffusion can differentiate sCJD from other forms of progressive dementia.

\section{Biomarkers in CSF}

Several CSF biomarkers were proposed to play a significant role in the diagnosis of sCJD. The most commonly studied is 14-3-3 protein, which is a surrogate marker for CJD and appears after neuronal destruction. Although there is still some debate in the literature, several studies conclude that the sensitivity of positive 14-3-3 protein in CSF for classic sCJD is $92 \%-96 \% .^{50}$ Tau protein is also 
released with damage to neurons, and in classic sCJD has a sensitivity of $81 \%$ and a specificity of $85 \%$; when tested in combination with $14-3-3$ protein the positive predictive value is $91 \% .^{9}$ This is in contrast to the lower sensitivity of $53 \%$ in vCJD. ${ }^{5,6,18}$

The timing of CSF testing in relation to disease stage is crucial as well. Both 14-3-3 protein and tau protein are biomarkers for rapid neuronal destruction, and so the total concentrations increase as CJD progresses. When looking at the sensitivities and specificities of these tests in the course of the disease, Chohan et al. found a lower sensitivity and specificity of $64 \%$ and $71 \%$, respectively, in the first third of the disease duration compared with $91 \%$ and $83 \%$ in the final third stage. ${ }^{12}$ The clinical picture is a necessary context when sending off for these studies. In the absence of clinical presentation, 14-3-3 detection has little to no value, because these results can be positive in Alzheimer disease or other disorders resulting in neuronal damage. ${ }^{5,6}$ Given the nonspecificity of these biomarkers, they should only be used to give confidence in the diagnosis with the right clinical picture, and never used to rule out the disease.

The problem with using these biomarkers is that they are not specific for CJD, because they are also found in many other diseases of the brain in which the pathology is neuronal loss. Positive 14-3-3 results are seen in viral encephalitides, recent stroke, subarachnoid hemorrhage, hypoxic brain damage, metabolic encephalopathy, glioblastoma, carcinomatous meningitis, paraneoplastic disorders, and corticobasal degeneration. ${ }^{57}$

Real-time quaking-induced conversion (RT-QuIC) is a recently described laboratory technique that provides definitive diagnosis of CJD from CSF samples by detecting $\mathrm{PrPSc}^{\mathrm{S}}$. The RT-QuIC technique uses recombinant $\mathrm{PrP}$ as a substrate that is seeded with $\mathrm{PrP}^{\mathrm{Sc}}$ and undergoes intermittent automated shaking, which amplifies the small amount of protein present into an accumulation of amyloid fibrils. Thioflavin $\mathrm{T}$ binds to these fibrils, and emits fluorescence that is detected in real time. ${ }^{1}$ The test has $80 \%-90 \%$ sensitivity, but is better than the test for 14-3-3 protein because it is $100 \%$ specific. ${ }^{34}$ There is also no difference in detection among the genetic subtypes.

As this methodology improves and becomes widely available, the test for 14-3-3 protein will become obsolete. The samples used in the test are becoming easier to obtain as well. Orrú et al. used RT-QuIC with nasal brushings and showed a sensitivity of $97 \%$ and specificity of $100 \%$, with positive results seen within 30 hours. These impressive results may be due to the suspicion that prions are shed in the olfactory nerves. This method is even less invasive than lumbar puncture, which only had $77 \%$ sensitivity when the CSF was tested in the same patients. ${ }^{39}$ Therefore, this modern technique should be part of the standard, initial testing for CJD, and part of updated diagnostic criteria for the disease.

\section{Treatment}

Despite all the advances in the understanding of this disease, the prognosis remains grim-CJD is a terminal disease. The mainstay of treatment is symptomatic and supportive, for example, using clonazepam for the treatment of myoclonus. Otto et al. showed a statistically significant improvement in cognitive function in a group of 28 patients with CJD treated with flupirtine, but this is the only study in the literature to report any symptom improvement with the use of medication. ${ }^{40}$ Future targets of therapy involve preventing the conversion of $\mathrm{PrP}^{\mathrm{C}}$ to $\mathrm{PrP}^{\mathrm{Sc}}$.

\section{Epidemiology}

To evaluate the age-related incidence of CJD in the US, we queried the National (Nationwide) Inpatient Sample (NIS) (https://www.hcup-us.ahrq.gov/nisoverview.jsp $)^{49}$ to identify age-related frequency of inpatient hospitalization for CJD (ICD-9-CM diagnosis code 046.1, 046.11, and 046.19) between 2002 and 2011. Using dischargelevel weights (DISCWT), 4428 cases were identified as CJD. The highest proportion of cases were observed in the 65 - to 69 -year-old group $(820 ; 18.52 \%)$, followed by $70-74$ years $(629 ; 14.21 \%)$ and $75-79$ years $(625 ; 14.11 \%)$, followed by a decline (Fig. 1). Although limitations of the NIS are well known, including coding errors, these numbers approximately represent the epidemiology of inpatient admissions for CJD.

A comparison of incurred mean hospital charges for the inpatient stay for those undergoing brain biopsy/surgery found that costs were significantly higher than in those without any surgical intervention $(\mathrm{p}<0.0001)$ for the years 2003-2008 and 2010-2011 (Fig. 2).

\section{Discussion}

Creuztfeldt-Jakob disease started out as a description of unusual neuropathological and clinical findings associated with other diseases in a series of 6 patients; over the last 90 years research has led to a well-described clinical and pathological entity, with typical imaging characteristics. The laboratory study of the disease also led to the development of new research techniques. More importantly, the study of prions demonstrated a novel disease pathogenesis devoid of DNA, dependent on pathological protein accumulation for propagation, and opened new fields of research in molecular and cellular biology, biochemistry, and biophysics that led to a Nobel Prize. ${ }^{43}$

\section{Need for New Diagnostic Criteria?}

Despite the wealth of new information at present, most clinicians are at a disadvantage in diagnosing the disease, given that the WHO guidelines are outdated. A physician faced with the challenging task of diagnosing a patient with progressive neurological decline may not be aware of all the information at hand and may request a brain biopsy without a thorough diagnostic workup. Likewise, neurosurgeons may proceed with a biopsy that, based on the literature, has a low probability of being diagnostic, and an even lower chance of making a difference in the patient's treatment plan or disease course. Newer diagnostic criteria do include hyperintensities on MRI as one of the tests that can be positive to make the case for probable CJD, but this still does not take into account more recent advances in research on the disease. Criteria for a diagnosis of CJD that includes newer confirmatory tests and incorporates 


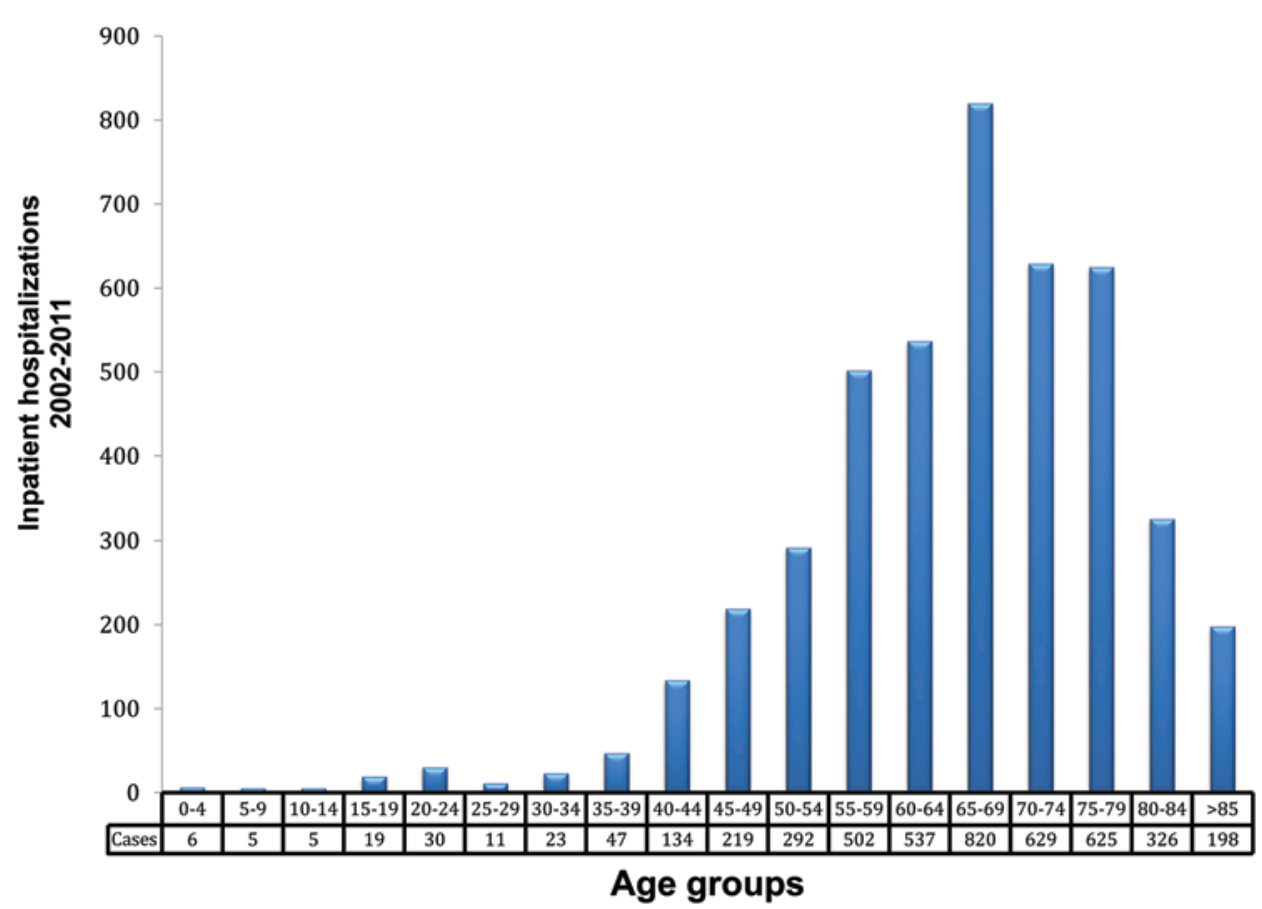

FIG. 1. Bar graph showing number of cases of CJD in the US, stratified by age group, over a 10-year period from the NIS.

phenotypic strain provide a less invasive means of definitive diagnosis and will include the atypical patient. An updated diagnostic criteria and algorithm should provide confidence in the diagnosis, while avoiding unnecessary costs, waste of resources, and the potential morbidity associated with a biopsy.

\section{Proposal for a Diagnostic Algorithm}

The best approach to a suspected case of CJD is with a critical appraisal of the information known about the disease. We now have a classification of the different sub-

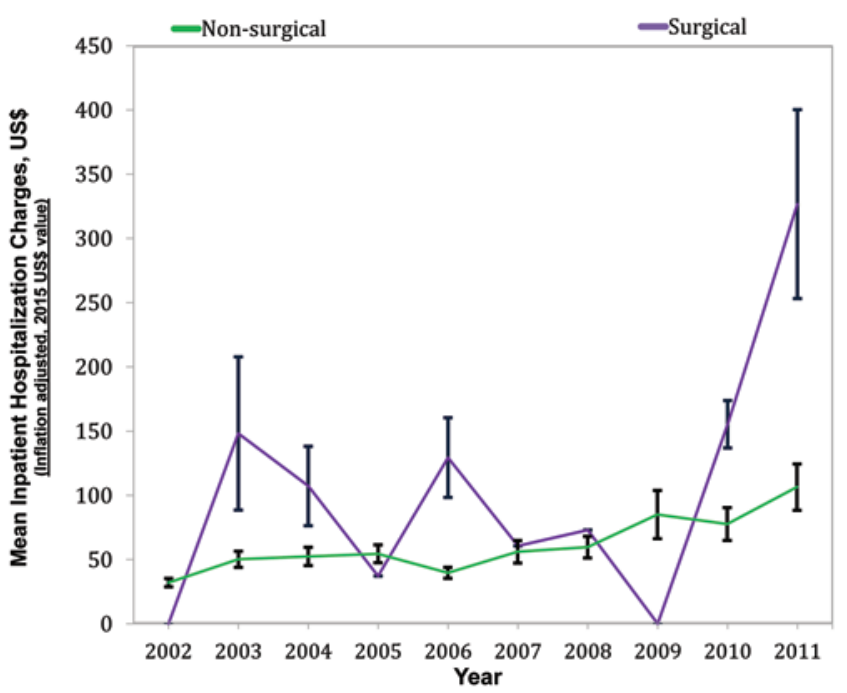

FIG. 2. Graph showing a comparison of total hospitalization costs between patients with CJD without surgery and those who did have a surgical procedure during their hospital stay. Data are taken from the NIS. types of disease present, and the goal in classifying prion diseases is to associate phenotype with genotype to aid diagnosis. If the most common imaging, clinical, and laboratory findings are studied in the context of the genotype, the clinician will have less doubt about the diagnosis. This is one more factor to be taken into account in updated diagnostic criteria. An updated list of criteria (Table 4) is proposed that takes into account CDI and RT-QuIC as part of the definitive diagnostics, removes the stipulation that the disease be present for less than 2 years (there are many reports in the literature of slowly progressive variants with a clinical course longer than 24 months), and includes specific phenotypes of the less common molecular strains.

An algorithm for the initial workup is provided in Fig. 3. The RT-QuIC technique is the most sensitive and specific antemortem diagnostic test, and should be the first one performed in the workup of a patient with suspected CJD, because it is the only study needed to diagnose the disease. A positive result will save money and resources by negating the need for any other studies. Because this test may not be available to many facilities yet, the proposed diagnostic pathway is for those without access to RT-QuIC, or for whom the results are negative.

This algorithm places diagnostic tests in order of sensitivity and timing of the disease course. The 14-3-3 protein is tested first, because CSF will be sent off to diagnose potentially treatable disorders. The routine CSF studies of glucose, total protein, white blood cell count, total cell count, and oligoclonal IgG in patients with CJD are generally unremarkable (Stoeck et al. ${ }^{50}$ ). This is of the utmost importance, because most other diseases in the differential diagnosis will usually have some abnormal result. Vasculitis, encephalitis, autoimmune disorders, and demyelination all usually have either CSF pleocytosis or elevated 
TABLE 4. Proposed updated diagnostic criteria for CJD*

\begin{tabular}{|c|c|c|c|}
\hline A & B & C & $\mathrm{D}$ \\
\hline \multirow{8}{*}{$\begin{array}{l}\text { Rapidly progres- } \\
\text { sive dementia }\end{array}$} & 1) Myoclonus & \multirow[t]{3}{*}{ PSWCs on EEG } & \multirow{8}{*}{$\begin{array}{l}\text { Routine investigations } \\
\text { that do not indicate an } \\
\text { alternative diagnosis }\end{array}$} \\
\hline & 2) Visual or cerebellar signs & & \\
\hline & 3) Pyramidal/extrapyramidal signs & & \\
\hline & 4) Akinetic mutism & \multirow[t]{3}{*}{ Positive 14-3-3 CSF assay } & \\
\hline & 5) VV2 genotype w/ predominant ataxia w/o myoclonus in early stage & & \\
\hline & $\begin{array}{l}\text { 6) MV2 genotype w/ long disease course }(>12 \text { mos) \& predominant } \\
\text { ataxia in early stage }\end{array}$ & & \\
\hline & $\begin{array}{l}\text { 7) MM2 genotype \& temporal lobe changes on MRI w/o basal ganglia } \\
\text { involvement }\end{array}$ & \multirow{2}{*}{$\begin{array}{l}\text { MRI high-intensity signal } \\
\text { abnormalities in caudate } \\
\text { nucleus \&/or putamen on } \\
\text { DW or FLAIR imaging }\end{array}$} & \\
\hline & $\begin{array}{l}\text { 8) VV1 genotype \& young age at onset (<50 yrs), slowly progressive } \\
\text { disease w/ frontotemporal dementia }\end{array}$ & & \\
\hline
\end{tabular}

* Probable $C J D=(A)+$ at least 2 of $(B$ [criteria 1-4]) + positive result on at least 1 of $(C)+(D)$. Possible $C J D=(A)+$ at least 2 of $(B)+$ absent $(C)+$ absent $(D)$. Definite $\mathrm{CJD}$ = diagnosed by standard neuropathological techniques; and/or immunocytochemically; and/or CDI-confirmed PrP; and/or positive RT-QulC of CSF or nasal brushings.

protein levels, and should be ruled out in the initial round of testing. MRI is more sensitive than EEG and should be done next if there are negative results on the 14-3-3 assay, and then EEG is last because it is the least sensitive test of the three. An MRI study that shows the described CJD findings in combination with the usual symptomatology points toward CJD, and PSWCs are highly specific as well, so a positive test provides confidence in the diagnosis of probable CJD.

An examination finding in conjunction with genetic testing increases the diagnostic assuredness of the physician. Cerebellar signs or visual changes are atypical examination findings that are typical in CJD, and so a good history of the present illness is vital. In the patient with akinetic mutism and myoclonus, CJD should be at the top of the differential diagnosis. Those presenting with ataxia as the predominant symptom and MV1 or VV2 subtypes strengthen the probability of CJD. A long disease course with prominent ataxia and an MV2 genotype should give confidence in the CJD diagnosis, whereas a patient with temporal lobe hyperintensity on MRI, without ataxia, and an MM2 genotype also should be considered a probable case of CJD. On the other hand, a young patient $(<50$ years) is likely to have the VV1 type.

\section{Utility of Biopsy}

The gold standard for diagnosis is pathological confirmation from a brain biopsy, but the frequency of a positively diagnostic biopsy is surprisingly low. Bai et al. performed a meta-analysis of 20 studies that described brain biopsy in cryptogenic neurological disease, and found an average diagnostic success rate of $54 \%$, with a morbidity of 9\%. ${ }^{2}$ Twenty-six biopsies at a CJD surveillance unit in Germany, where all suspected cases of CJD are referred, showed that $42 \%$ of the biopsies were nondiagnostic. ${ }^{20}$ All patients with CJD lacked inflammatory CSF numbers, and there was a statistically significant difference in the presence of myoclonus in CJD patients (82\%) versus those with another disease (27\%), again highlighting the necessity of accounting for all clinical information.

\section{Tissue Confirmatory Tests}

The reason for the low diagnostic yield of brain biopsy is the methodology used in the pathological sections. Histopathological investigation must show vacuolation, which may be missed on specimens. There needs to be ample amyloid deposition present for a positive immunohistochemical test for PrP. The CDI is a newer method that can detect the disease with a better diagnostic success rate than the current tests. Safar et al. used the CDI to examine 8 different regions of the brain in 28 patients who died of CJD. These investigators found 100\% accuracy and detection of $\mathrm{PrP}^{\mathrm{Sc}}$ in all regions tested in all patients, and no false positives in the control group. This is dramatically better than the diagnostic sensitivity of $17 \%$ and $22 \%$ for histological and immunohistochemical tests, respectively, in the comparison group. ${ }^{45}$ Another advantage of the CDI is that any part of the brain may be tested; it does not need to be an area with maximal change on imaging studies, and so a nondominant frontal lobe tissue sample will suffice.

\section{Indication for Biopsy}

The argument against biopsy in CJD is that not only are the diagnostic success rates low, but more importantlywill this lead to any change in treatment plan or disease course? In a study of 90 patients with rapidly progressive dementia, Schott et al. showed a 57\% diagnostic rate, but only $11 \%$ of these diagnoses resulted in a change of treatment. ${ }^{46}$ Some will argue that because a biopsy will provide no change in the treatment, it only is adding costs and wasting resources that can be used elsewhere. Figure 2 shows a recent trend in hospital costs associated with those undergoing a surgical procedure that provides no benefit. It is important for treating or consulting physicians to know that the RT-QuIC test exists and that getting brain tissue is unnecessarily invasive and has a lower diagnostic sensitivity.

Proponents of brain biopsy argue that the main justification for biopsy is to diagnose a potentially treatable disease or confirm the diagnosis of CJD in the presence 


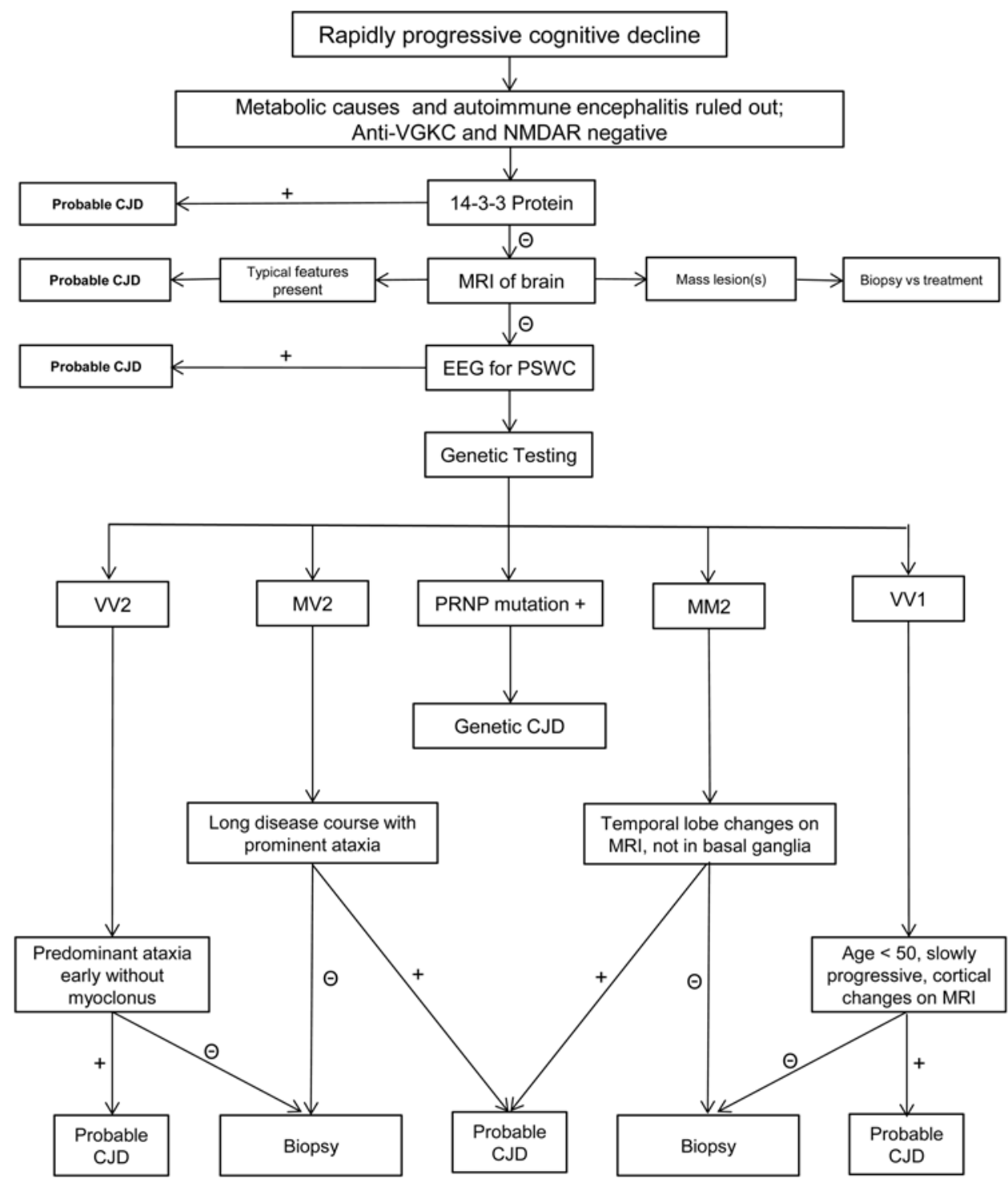

FIG. 3. Proposed diagnostic pathway for the workup of a patient with rapidly progressive cognitive decline. NMDAR = N-methyl-Daspartate receptor; VGKC = voltage-gated potassium channel.

of atypical features. These include CNS vasculitis, encephalitis (viral or autoimmune), malignancy, demyelination, nonviral CNS infections, and sarcoidosis. Schuette et al. showed a rate of change of $8 \%$ in the treatment plan in a series of 135 consecutive brain biopsies, and in only $4 \%$ did the findings lead to an improvement in the disease course. ${ }^{47}$ Chitravas et al. looked at 1106 patients who underwent a brain biopsy for dementia and found that only $6 \%$ of the patients in the study had a treatable disorder (i.e., not CJD) and that two-thirds of these cases did not meet WHO criteria for CJD. ${ }^{11}$

If all patient information is taken into account, then the likelihood of missing a treatable disease on brain biopsy is very low. Neoplasms are often the most common conditions found in these studies, but MRI would easily differentiate between the two, because prion diseases do not present with mass lesions or gadolinium uptake. History, physical examination, MRI, and routine CSF studies are ample and necessary to separate treatable conditions (that may need a brain biopsy) from prion disease, which can be confirmed in less invasive ways.

Brain biopsy should only be done after all noninvasive diagnostic avenues are exhausted, and do not point to a cause. Because a biopsy for dementia is rare, there is no way to standardize when to perform the procedure. All information must be taken into account: the prominent clinical signs, patient's age, duration of disease, imaging studies, EEG results, and RT-QuIC assay of either nasal brushing or CSF. In the patient with atypical features, no findings on imaging studies or EEG, and negative CSF studies (including ruling out the autoimmune and viral encephalitides), a biopsy is indicated and the CDI must be the diagnostic test used.

\section{Conclusions}

Creutzfeldt-Jakob disease is a fatal neurological condi- 
tion that often perplexes the treating physician. Given its rarity, published diagnostic criteria aid in the approach to diagnosis, but these are unfortunately outdated and do not incorporate modern research laboratory methods. The proposed updated diagnostic criteria and treatment algorithm are based on the latest research, and help guide the workup in a disease that can be difficult to diagnose. Knowledge of different strain phenotypes is crucial to making an accurate diagnosis, and a brain biopsy need be performed only rarely in the atypical patient, because RT-QuIC appears to be the best test to provide a confirmed diagnosis.

\section{Acknowledgments} port.

We thank Drs. Osama Ahmed and Tanmoy Maiti for their sup-

\section{References}

1. Atarashi R, Satoh K, Sano K, Fuse T, Yamaguchi N, Ishibashi $\mathrm{D}$, et al: Ultrasensitive human prion detection in cerebrospinal fluid by real-time quaking-induced conversion. Nat Med 17:175-178, 2011

2. Bai HX, Zou Y, Lee AM, Lancaster E, Yang L: Diagnostic value and safety of brain biopsy in patients with cryptogenic neurological disease: a systematic review and meta-analysis of 831 cases. Neurosurgery 77:283-295, 2015

3. Barboriak DP, Provenzale JM, Boyko OB: MR diagnosis of Creutzfeldt-Jakob disease: significance of high signal intensity of the basal ganglia. AJR Am J Roentgenol 162:137-140, 1994

4. Boëlle PY, Cesbron JY, Valleron AJ: Epidemiological evidence of higher susceptibility to vCJD in the young. BMC Infect Dis 4:26, 2004

5. Brown P, Brunk C, Budka H, Cervenakova L, Collie D, Green A, et al: WHO Manual for Surveillance of Human Transmissible Spongiform Encephalopathies, including Variant Creutzfeldt-Jakob Disease. Geneva: World Health Organization, Communicable Disease Surveillance and Response, 2003

6. Brown P, Preece M, Brandel JP, Sato T, McShane L, Zerr I, et al: Iatrogenic Creutzfeldt-Jakob disease at the millennium. Neurology 55:1075-1081, 2000

7. Büeler H, Fischer M, Lang Y, Bluethmann H, Lipp HP, DeArmond SJ, et al: Normal development and behaviour of mice lacking the neuronal cell-surface PrP protein. Nature 356:577-582, 1992

8. Caobelli F, Cobelli M, Pizzocaro C, Pavia M, Magnaldi S, Guerra UP: The role of neuroimaging in evaluating patients affected by Creutzfeldt-Jakob disease: a systematic review of the literature. J Neuroimaging 25:2-13, 2015

9. Castellani RJ, Colucci M, Xie Z, Zou W, Li C, Parchi P, et al: Sensitivity of 14-3-3 protein test varies in subtypes of sporadic Creutzfeldt-Jakob disease. Neurology 63:436-442, 2004

10. Centers for Disease Control and Prevention: CreutzfeldtJakob disease associated with cadaveric dura mater grafts -- Japan, January 1979-May 1996. MMWR Morb Mortal Wkly Rep 46:1066-1069, 1997

11. Chitravas N, Jung RS, Kofskey DM, Blevins JE, Gambetti P, Leigh RJ, et al: Treatable neurological disorders misdiagnosed as Creutzfeldt-Jakob disease. Ann Neurol 70:437-444, 2011

12. Chohan G, Pennington C, Mackenzie JM, Andrews M, Everington D, Will RG, et al: The role of cerebrospinal fluid 14-33 and other proteins in the diagnosis of sporadic CreutzfeldtJakob disease in the UK: a 10-year review. J Neurol Neurosurg Psychiatry 81:1243-1248, 2010

13. Collie DA, Summers DM, Sellar RJ, Ironside JW, Cooper S,
Zeidler M, et al: Diagnosing variant Creutzfeldt-Jakob disease with the pulvinar sign: MR imaging findings in 86 neuropathologically confirmed cases. AJNR Am J Neuroradiol 24:1560-1569, 2003

14. Creutzfeldt H: Uber eine eigenartige herdformige Erkrankung des Zentralnervensystems. Z Gesamte Neurol Psychiatr 57:1-18, 1920

15. Duffy P, Wolf J, Collins G, DeVoe AG, Streeten B, Cowen D: Possible person-to-person transmission of Creutzfeldt-Jakob disease. N Engl J Med 290:692-693, 1974 (Letter)

16. Gambetti P, Kong Q, Zou W, Parchi P, Chen SG: Sporadic and familial CJD: classification and characterisation. $\mathbf{B r}$ Med Bull 66:213-239, 2003

17. Gao T, Lyu JH, Zhang JT, Lou X, Zhao W, Xing XW, et al: Diffusion-weighted MRI findings and clinical correlations in sporadic Creutzfeldt-Jakob disease. J Neurol 262:14401446, 2015

18. Green A, Sanchez-Juan P, Ladogana A, Cuadrado-Corrales N, Sánchez-Valle R, Mitrová E, et al: CSF analysis in patients with sporadic CJD and other transmissible spongiform encephalopathies. Eur J Neurol 14:121-124, 2007

19. Head MW: Human prion diseases: molecular, cellular and population biology. Neuropathology 33:221-236, 2013

20. Heinemann U, Krasnianski A, Meissner B, Kallenberg K, Kretzschmar HA, Schulz-Schaeffer W, et al: Brain biopsy in patients with suspected Creutzfeldt-Jakob disease. J Neurosurg 109:735-741, 2008

21. Hilton DA: Pathogenesis and prevalence of variant Creutzfeldt-Jakob disease. J Pathol 208:134-141, 2006

22. Jakob A: Uber eigenartige Erkrankungen des Zentralnervensystems mit bemerkenswerten anatomischen Befunde (spastische Pseudosklerose-Encephalomyelopathie mit dissminierten Degeneratiosherden). Z Ges Neurol Psychiatry 64:147-228, 1921

23. Johnson RT, Gibbs CJ Jr: Creutzfeldt-Jakob disease and related transmissible spongiform encephalopathies. N Engl J Med 339:1994-2004, 1998

24. Kallenberg K, Schulz-Schaeffer WJ, Jastrow U, Poser S, Meissner B, Tschampa HJ, et al: Creutzfeldt-Jakob disease: comparative analysis of MR imaging sequences. AJNR Am J Neuroradiol 27:1459-1462, 2006

25. Knight RS, Will RG: Prion diseases. J Neurol Neurosurg Psychiatry 75 (Suppl 1):i36-i42, 2004

26. Krasnianski A, Meissner B, Schulz-Schaeffer W, Kallenberg $\mathrm{K}$, Bartl M, Heinemann U, et al: Clinical features and diagnosis of the MM2 cortical subtype of sporadic CreutzfeldtJakob disease. Arch Neurol 63:876-880, 2006

27. Krasnianski A, Schulz-Schaeffer WJ, Kallenberg K, Meissner B, Collie DA, Roeber S, et al: Clinical findings and diagnostic tests in the MV2 subtype of sporadic CJD. Brain 129:2288-2296, 2006

28. Lee H, Cohen OS, Rosenmann H, Hoffmann C, Kingsley PB, Korczyn AD, et al: Cerebral white matter disruption in Creutzfeldt-Jakob disease. AJNR Am J Neuroradiol 33:1945-1950, 2012

29. Liao YC, Lebo RV, Clawson GA, Smuckler EA: Human prion protein cDNA: molecular cloning, chromosomal mapping, and biological implications. Science 233:364-367, 1986

30. Llewelyn CA, Hewitt PE, Knight RS, Amar K, Cousens $\mathrm{S}$, Mackenzie J, et al: Possible transmission of variant Creutzfeldt-Jakob disease by blood transfusion. Lancet 363:417-421, 2004

31. Mahale RR, Javali M, Mehta A, Sharma S, Acharya P, Srinivasa R: A study of clinical profile, radiological and electroencephalographic characteristics of suspected Creutzfeldt-Jakob disease in a tertiary care centre in South India. J Neurosci Rural Pract 6:39-50, 2015

32. Maheshwari A, Fischer M, Gambetti P, Parker A, Ram A, Soto C, et al: Recent US Case of Variant Creutzfeldt-Jakob 
Disease-Global Implications. Emerg Infect Dis 21:750-759, 2015

33. Masters CL: Creutzfeldt-Jakob disease: its origins. Alzheimer Dis Assoc Disord 3:46-51, 1989

34. McGuire LI, Peden AH, Orrú CD, Wilham JM, Appleford NE, Mallinson G, et al: Real time quaking-induced conversion analysis of cerebrospinal fluid in sporadic CreutzfeldtJakob disease. Ann Neurol 72:278-285, 2012

35. Mead S: Prion disease genetics. Eur J Hum Genet 14:273281, 2006

36. Meissner B, Westner IM, Kallenberg K, Krasnianski A, Bartl M, Varges D, et al: Sporadic Creutzfeldt-Jakob disease: clinical and diagnostic characteristics of the rare VV1 type. Neurology 65:1544-1550, 2005

37. Na DL, Suh CK, Choi SH, Moon HS, Seo DW, Kim SE, et al: Diffusion-weighted magnetic resonance imaging in probable Creutzfeldt-Jakob disease: a clinical-anatomic correlation. Arch Neurol 56:951-957, 1999

38. Nevin S, McMenemey WH, Behrman S, Jones DP: Subacute spongiform encephalopathy-a subacute form of encephalopathy attributable to vascular dysfunction (spongiform cerebral atrophy). Brain 83:519-564, 1960

39. Orrú CD, Bongianni M, Tonoli G, Ferrari S, Hughson AG, Groveman BR, et al: A test for Creutzfeldt-Jakob disease using nasal brushings. N Engl J Med 371:519-529, 2014

40. Otto M, Cepek L, Ratzka P, Doehlinger S, Boekhoff I, Wiltfang J, et al: Efficacy of flupirtine on cognitive function in patients with CJD: A double-blind study. Neurology 62:714718, 2004

41. Parchi P, Giese A, Capellari S, Brown P, Schulz-Schaeffer W, Windl O, et al: Classification of sporadic Creutzfeldt-Jakob disease based on molecular and phenotypic analysis of 300 subjects. Ann Neurol 46:224-233, 1999

42. Parchi P, Saverioni D: Molecular pathology, classification, and diagnosis of sporadic human prion disease variants. Folia Neuropathol 50:20-45, 2012

43. Prusiner SB: Novel proteinaceous infectious particles cause scrapie. Science 216:136-144, 1982

44. Prusiner SB: Prions. Proc Natl Acad Sci U S A 95:1336313383, 1998

45. Safar JG, Geschwind MD, Deering C, Didorenko S, Sattavat M, Sanchez H, et al: Diagnosis of human prion disease. Proc Natl Acad Sci U S A 102:3501-3506, 2005

46. Schott JM, Reiniger L, Thom M, Holton JL, Grieve J, Brandner $\mathrm{S}$, et al: Brain biopsy in dementia: clinical indications and diagnostic approach. Acta Neuropathol 120:327-341, 2010

47. Schuette AJ, Taub JS, Hadjipanayis CG, Olson JJ: Open biopsy in patients with acute progressive neurologic decline and absence of mass lesion. Neurology 75:419-424, 2010

48. Spielmeyer W: Die histopathologische Forschung in der Psychiatrie. Klin Wochenschr 1:1817-1819, 1922

49. Steiner C, Elixhauser A, Schnaier J: The healthcare cost and utilization project: an overview. Eff Clin Pract 5:143-151, 2002

50. Stoeck K, Sanchez-Juan P, Gawinecka J, Green A, Lado- gana A, Pocchiari M, et al: Cerebrospinal fluid biomarker supported diagnosis of Creutzfeldt-Jakob disease and rapid dementias: a longitudinal multicentre study over 10 years. Brain 135:3051-3061, 2012

51. Traub RD, Pedley TA: Virus-induced electrotonic coupling: hypothesis on the mechanism of periodic EEG discharges in Creutzfeldt-Jakob disease. Ann Neurol 10:405-410, 1981

52. United States Public Health Service: Public Health Service Interagency Coordinating Committee Report on Human Growth Hormone and CJD. Washington, DC: US Public Health Service, 1997, Vol 14, pp 1-11

53. Vitali P, Maccagnano E, Caverzasi E, Henry RG, Haman A, Torres-Chae C, et al: Diffusion-weighted MRI hyperintensity patterns differentiate CJD from other rapid dementias. Neurology 76:1711-1719, 2011

54. Wieser HG, Schindler K, Zumsteg D: EEG in CreutzfeldtJakob disease. Clin Neurophysiol 117:935-951, 2006

55. Wieser HG, Schwarz U, Blättler T, Bernoulli C, Sitzler M, Stoeck K, et al: Serial EEG findings in sporadic and iatrogenic Creutzfeldt-Jakob disease. Clin Neurophysiol 115:24672478,2004

56. Will RG, Ironside JW, Zeidler M, Cousens SN, Estibeiro K, Alperovitch A, et al: A new variant of Creutzfeldt-Jakob disease in the UK. Lancet 347:921-925, 1996

57. World Health Organization: Global Surveillance, Diagnosis, and Therapy of Human Transmissible Spongiform Encephalopathies: Report of a WHO Consultation. Geneva: World Health Organization, 1998

58. Zerr I, Schulz-Schaeffer WJ, Giese A, Bodemer M, Schröter A, Henkel K, et al: Current clinical diagnosis in CreutzfeldtJakob disease: identification of uncommon variants. Ann Neurol 48:323-329, 2000

\section{Disclosure}

The authors report no conflict of interest concerning the materials or methods used in this study or the findings specified in this paper.

\section{Author Contributions}

Conception and design: Manix, Henry, Guthikonda. Acquisition of data: Manix, Kalakoti, Thakur, Menger. Analysis and interpretation of data: Manix, Kalakoti, Thakur, Menger. Drafting the article: Manix, Kalakoti, Henry. Critically revising the article: Manix, Henry, Thakur, Menger, Guthikonda. Reviewed submitted version of manuscript: Manix, Kalakoti, Henry, Guthikonda. Administrative/technical/material support: Nanda, Kalakoti. Study supervision: Nanda, Guthikonda.

\section{Correspondence}

Anil Nanda, Department of Neurosurgery, Louisiana State University Health Sciences Center-Shreveport, 1501 Kings Hwy., Shreveport, LA 71130. email: ananda@lsuhsc.edu. 